\title{
Dementia Care Mapping (DCM): initial validation of DCM 8 in UK field trials
}

\author{
Dawn J. Brooker* and Claire Surr \\ University of Bradford, Bradford, UK
}

\section{SUMMARY}

Objectives This paper describes DCM 8 and reports on the initial validation study of DCM 8 .

Methods Between 2001-2003, a series of international expert working groups were established to examine various aspects of DCM with the intention of revising and refining it. During 2004-2005 the revised tool (DCM 8) was piloted in seven service settings in the UK and validated against DCM 7th edition.

Results At a group score level, WIB scores and spread of Behavioural Category Codes were very similar, suggesting that group scores are comparable between DCM 7 and 8. Interviews with mappers and focus groups with staff teams suggested that DCM 8 was preferable to DCM 7th edition because of the clarification and simplification of codes; the addition of new codes relevant to person-centred care; and the replacement of Positive Events with a more structured recording of Personal Enhancers.

Conclusions DCM 8 appears comparable with DCM 7th edition in terms of data produced and is well received by mappers and dementia care staff. Copyright (C) 2006 John Wiley \& Sons, Ltd.

KEY WORDS - dementia; DCM; DCM 8; person centred care; quality of life; quality of care

\section{INTRODUCTION}

Dementia Care Mapping (DCM) (Bradford Dementia Group, 1997, 2005) is an observational tool that has been used in formal dementia care settings since 1992, both as an instrument for developing person-centred care practice, and as a tool in evaluative research (Brooker, 2005). DCM 7th Edition has been described fully elsewhere (Kuhn et al., 2000; Brooker et al., 2004). In brief, an observer (mapper) observes five people with dementia (participants) continuously over a representative time period in communal areas of care facilities. After each 5-min period (a time-frame) two types of codes are used to record what has happened to each participant. The Behavioural Category Code (BCC) describes one of 24 different domains of participant behaviour that has occurred. The mapper also makes a decision for each time frame, based on behavioural

\footnotetext{
*Correspondence to: Prof. D. J. Brooker, Bradford Dementia Group, School of Health Studies, University of Bradford, Unity Building, 25 Trinity Road, Bradford. BD5 0BB UK.

E-mail: d.j.brooker@bradford.ac.uk
}

Copyright (C) 2006 John Wiley \& Sons, Ltd. indicators, about the relative state of ill-being or wellbeing experienced by the person with dementia, called a Well/Ill Being (WIB) Value. This is expressed on a sixpoint scale ranging from extreme ill-being $(-5)$ to extreme well-being $(+5)$. Level of well-being and illbeing is decided upon according to a number of behavioural indicators. The WIB values attached to some BCCs, however, such as sleeping or withdrawn behaviours, degenerate as a function of time. This 'degeneration rule' as it is called, means that in BCCs such as $\mathrm{C}$ (withdrawn behaviour) which would receive a WIB value of -1 , would degenerate to -3 if it was allowed to continue for six 5-min time-frames, and to -5 after a further six uninterrupted time-frames. Using the degeneration rule, there does not need to be an accompanying overt indication of a worsening of ill-being.

WIB values can be averaged to arrive at a WIB score. This provides an index of relative well-being for a particular time period for an individual or a group. In addition, staff behaviours known as Personal Detractions (PDs) and Positive Events (PEs) are recorded whenever they occur. Personal Detractions are staff behaviours that have the potential to undermine the personhood of those with dementia (Kitwood, 1997). These are described and 
coded according to type and severity. Positive Events are recorded in qualitative notes by the mapper but these are not coded in a systematic manner.

The method and coding system were originally developed through ethological observations of many hours in nursing homes, hospital facilities and daycare in the UK (Kitwood and Bredin, 1994). DCM has been through a number of changes since its inception. DCM 7 was introduced in 1997. There were, however, no published papers demonstrating the relationship between this and earlier editions.

Studies presenting research into the psychometric properties of DCM 7 have been published. In terms of concurrent validity there is evidence that DCM is related to indicators of quality of care such as incidence of pressure sores (Bredin et al., 1995) and other audits of service quality (Brooker et al., 1998). There is also evidence of concurrent validity of DCM WIB scores with proxy quality of life measures (Fossey et al., 2002; Edelman et al., 2005). Fossey et al. (2002) demonstrated test-retest reliability and internal consistency. Published research suggests it is possible to achieve acceptable inter-rater reliability with DCM 7 (Brooker, 2005). When many different mappers are engaged in mapping at different points in time, drifts in coding can have a significant impact on results (Thornton et al., 2004). For example one of the coding rules states that if sleeping occurs in the same time-frame as communication, then the communication is what is coded rather than sleep, even if the communication was only very brief compared to time spent asleep. If this rule is not applied consistently by all mappers, then the results will be markedly less favourable. Systematic checking by regularly ensuring interrater reliability to a gold-standard mapper can prevent this (Surr and Bonde-Nielsen, 2003).

DCM has been used for a variety of different purposes over the years. It was designed to be used in a series of developmental evaluations over time to help care teams identify ways in which they could improve the quality of person centred care. DCM was not designed as a research tool but has proved useful in this context. DCM has been used in cross-sectional surveys of quality of care and quality of life and to investigate the relationship between participant characteristics and well-being and activity. It has been used to evaluate a number of non-pharmacological therapeutic interventions and as part of the evaluation of larger scale changes in therapeutic regimen and organisation of care. It has also been used as an adjunct in multi-method qualitative evaluations. Readers are referred to a recent literature review
(Brooker, 2005) and to the University of Bradford bibliographic database on DCM (www.bradford.ac. uk/acad/health/dcm).

In 2001, an international 'Think Tank' of DCM practitioners came together to review their collective experience on DCM (Brooker and Rogers, 2001). This group identified a number of issues regarding DCM 7 that required attention. This paved the way for the subsequent revision to DCM 8. It identified inconsistencies between codes and unnecessary complexity in places. Degenerating WIB values as a function of time rather than as a change in overt behaviour was thought to contain many assumptions that were difficult to justify empirically. At an individual participant level, the range of Behaviour Category Codes was not thought in places to reflect current best practice models. In terms of quality of care, the lack of structure for positive event recording meant that important opportunities for feeding back on positive staff action were being lost. There was also a unanimous view that the way in which the tool was used was often as important as the tool itself in improving the quality of care for people with dementia. Guidance on how to get the best from the tool was not readily available. Since the development of DCM 7, DCM training is now available in seven countries world wide and DCM trained personnel are active in a further 20 countries. Much of the published research has also been undertaken in more recent years. The expertise in working with the tool has grown exponentially over the past seven years. This level of expertise is not available in the DCM 7 guidelines.

Between 2001-2003, a series of international working groups examined various aspects of DCM with the aim of revising and refining it. The remit was to develop a tool that was fit to be a driver for personcentred care within a wide range of care facilities.

This paper reports on the UK field trials for DCM 8 . Specifically the aims of the field trials were:

- To examine the concurrent validity of DCM 8 with DCM 7.

- To examine whether DCM 8 is practical to use.

- To assess whether DCM 8 provides useful feedback for staff to assess the quality of care they are providing, upon which to develop action plans for improving care on an individual and group level.

In addition this paper describes the DCM 8 tool.

\section{METHOD}

Study sites

Four care organisations were used for the field trials. The selection criteria were that they used DCM 7 
regularly as part of clinical governance, quality assurance, audit or best value review processes and that they could provide a range of different types of facility in which to conduct field trials. One or two facilities from each organisation were mapped as part of the field trials. The facilities were selected by the local DCM lead for the organisation.

\section{Measures}

$D C M$ 7. Within the field trials, DCM 7th Edition data was collected in strict adherence to the DCM 7th Edition manual (Bradford Dementia Group, 1997).

DCM 8. DCM 8 (Bradford Dementia Group, 2005; Surr, Brooker \& Edwards, 2006) has the same four basic coding frames as DCM 7. The majority of Behaviour Category Codes (BCC) have stayed the same, although all BCCs were re-written and the ambiguities between different codes clarified. Some DCM 7 codes have been re-labelled, some have been removed and some additional codes have been included. A list of DCM 8 BCCs appears in Table 1. Those that have changed significantly have been marked with an asterisk.

The six-point scale of -5 to +5 for observing well and ill being has been maintained but the criteria for determining $-5,-3,-1,+1,+3$ and +5 have been clarified. Well and ill being is a global state that cannot be determined by just one 5-min time-frame. What is now coded in the 5-min frame is an observation of the participants' mood state alongside their level of engagement with their environment. In DCM 8, the 5-min observation (formerly WIB value) has been renamed ME Value (mood/engagement value). These are shown in Table 2. Over the period of mapping, these ME values can be used to assess a WIB score (Well-ill being score) based on the preponderance of mood and engagement over time.

The operational rules for deciding what $\mathrm{BCC}$ to record when more than one occurs in the time-frame have been simplified. Degeneration of codes as a function of time, have been removed altogether. In DCM 8, N (Nod, sleep) is generally recorded without a ME value.

The 17 types of Personal Detraction have remained unchanged. In DCM 8, a major change is in the recording of Personal Enhancers. These have a parallel structure to Personal Detractions (Table 3). Personal Enhancers build on the description of positive person work (Kitwood, 1997). The PEs and PDs are further divided around the degree to which they support or undermine the five psychological needs described by Kitwood (1997).

For the purposes of the field trials, a draft DCM 8 manual was developed. This was used to guide all

Table 1. DCM 8 behaviour category codes

\begin{tabular}{lll}
\hline Code & Memory cue & General description of category \\
\hline A & Articulation & Interacting with others verbally or otherwise \\
$\mathrm{B}^{*}$ & Borderline & Being engaged but passively (watching) \\
$\mathrm{C}$ & Cool & Being disengaged, withdrawn \\
$\mathrm{D}^{*}$ & Doing for self & Self care \\
$\mathrm{E}$ & Expressive & Expressive or creative activities \\
$\mathrm{F}$ & Food & Eating or drinking \\
$\mathrm{G}^{*}$ & Going back & Reminiscence and life review \\
$\mathrm{I}$ & Intellectual & Prioritising the use of intellectual abilities \\
$\mathrm{J}$ & Joints & Exercise or physical sport \\
$\mathrm{K}$ & Kum and go & Walking, standing or moving independently \\
$\mathrm{L}^{*}$ & Leisure & Leisure, fun and recreational activities \\
$\mathrm{N}^{*}$ & Nod Land of & Sleeping, dozing \\
$\mathrm{O}^{*}$ & Objects & Displaying attachment to or relating to inanimate objects \\
$\mathrm{P}$ & Physical & Receiving practical, physical or personal care \\
$\mathrm{R}$ & Religion & Engaging in a religious activity \\
$\mathrm{S}$ & Sex & Sexual expression \\
$\mathrm{T}$ & Timalation & Direct engagement of the senses \\
$\mathrm{U}^{*}$ & Unresponded to & Attempting to communicate without receiving a response \\
$\mathrm{V}^{*}$ & Vocational & Work or work-like activity \\
$\mathrm{W}$ & Withstanding & Repetitive self-stimulation of a sustained nature \\
$\mathrm{X}$ & X-cretion & Episodes related to excretion \\
$\mathrm{Y}$ & Yourself & Interaction in the absence of any observable other \\
$\mathrm{Z}$ & Zero option & Fits none of existing categories \\
\hline
\end{tabular}

*significant changes from DCM 7. 
Table 2. DCM 8 scale of ME values

\begin{tabular}{lcl}
\hline Mood & ME value & \multicolumn{1}{c}{ Engagement } \\
\hline Very happy, cheerful. Very high positive mood. & +5 & Very absorbed, deeply engrossed/engaged. \\
Content, happy, relaxed. Considerable positive mood. & +3 & $\begin{array}{l}\text { Concentrating but distractible. Considerable engagement. } \\
\text { Alert and focussed on surroundings. Brief or } \\
\text { Neutral. Absence of overt signs of positive or }\end{array}$ \\
negative mood. & +1 & intermittent engagement. \\
Small signs of negative mood. & -1 & Withdrawn and out of contact. \\
Considerable signs of negative mood. & -3 & \\
Very distressed. Very great signs of negative mood. & -5 & \\
\hline
\end{tabular}

mapping decisions. DCM 8 mapping was carried out by the authors and a further three DCM trainers who all had a high level of expertise with the tool. Interrater reliability concordance of $70 \%$ was established between these expert mappers.

$C D S$. Dependency was measured by the Care Dependency Scale (Dijkstra et al., 2000). A higher score indicates a lower degree of dependency (max score $=80$ ).

\section{Procedures}

Following the initial identification and negotiation with the care organisation, the authors worked with the DCM lead in each organisation to manage the

Table 3. DCM 8 list of Personal Detractions and Enhancers

\begin{tabular}{lll}
\hline Personal Detraction Type & Personal Enhancer type \\
\hline & Comfort & \\
PD 1. Intimidation & & PE 1. Warmth \\
PD 2. Withholding & & PE 2. Holding \\
PD 3. Outpacing & PE 3. Relaxed pace \\
PD 4. Infantilazation & Identity & \\
PD 5. Labelling & & PE 4. Respect \\
PD 6. Disparagement & PE 5. Acceptance \\
& PE 6. Celebration \\
PD 7. Accusation & & PE 7. Acknowledgement \\
PD8. Treachery & & PE 8. Genuineness \\
PD 9. Invalidation & PE 9. Validation \\
PD 10. Disempowerment & & PE 10. Empowerment \\
PD 11. Imposition & & PE 11. Facilation \\
PD 12. Disruption & & PE 12. Enabling \\
PD 13. Objectification & & PE 13. Collaboration \\
PD 14. Stigmatization & Inclusion & \\
PD 15 Ignoring & & PE 14. Recognition \\
PD 16. Banishment & & PE 15. Including \\
PD 17. Mockery & PE16. Belonging \\
\hline
\end{tabular}

Copyright (C) 2006 John Wiley \& Sons, Ltd. evaluation in each facility, consisting of the following process:

1. Researchers briefed staff and service users about the research and sought initial consent in principle.

2. Inter-rater reliability of at least $70 \%$ agreement was established with local mapper(s) on DCM 7. Assent from participants' relatives and consent from participants and staff was established.

3. Researchers conducted a map using DCM 7 and 8 simultaneously lasting between 4-6 h. Local mappers observed the same participants simultaneously using DCM 7.

4. Data from DCM 7 and 8 were compared and results discussed with the local mappers.

5. A semi-structured interview was undertaken with the local mappers to assess their opinion of the DCM 8 and DCM 7 data.

6. Both the DCM 7 and DCM 8 data was fed back to the staff team and discussed.

7. A focus group was undertaken with the staff to gain their opinion on the usefulness of the DCM 8 data.

\section{RESULTS}

\section{Participants}

Thirty-nine people with dementia participated in the study. They were selected by the local mappers using their usual practice criteria. Table 4 shows different facilities, age, gender, number of time-frames and dependency data.

Thirty-five staff participated in the focus groups across the seven facilities. These included representatives from nursing, social work, occupational therapy, physiotherapy and clinical psychology.

\section{Concurrent validity between DCM 7 and 8}

Table 5 shows the WIB scores and BCC data from the concurrent mapping using DCM 7 and 8. The Group 


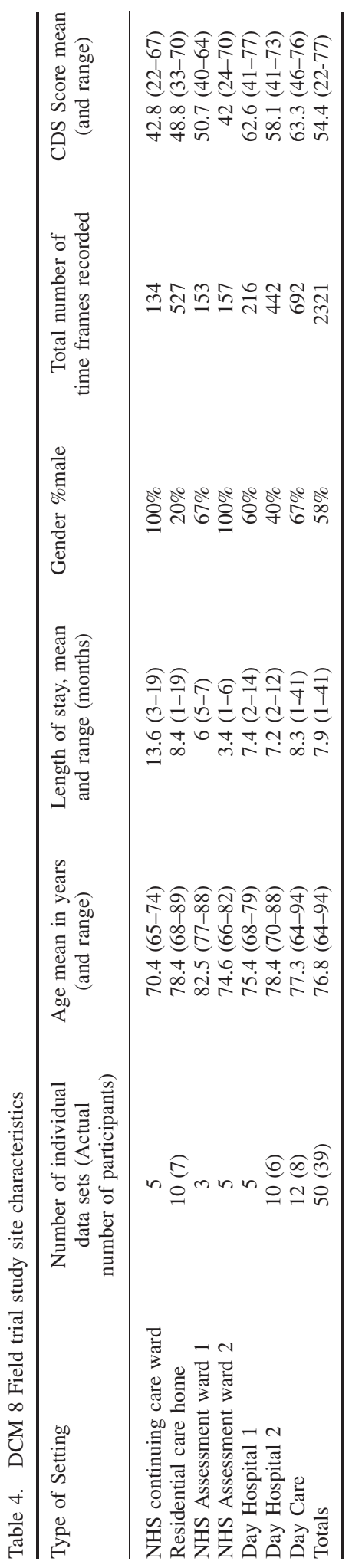

Copyright (C) 2006 John Wiley \& Sons, Ltd.



Int J Geriatr Psychiatry 2006; 21: 1018-1025. DOI: $10.1002 / \mathrm{gps}$ 
WIB scores and range of individual WIB scores are very similar and highly correlated. A Pearson Correlation coefficient of $0.974(p<0.0001)$ was calculated between the individual WIB scores on DCM 7 and 8.

Likewise the distribution of the WIB and ME values presented in Table 6 is very similar for DCM 7 and 8.

In both the DCM 7 and DCM 8 data, the top 5 BCCs accounted for around $75 \%$ (range $69-87 \%$ ) of the time-frames. The spread was very similar with only a few percentage points different. It is of interest to note that the new DCM 8 code L (Leisure) featured in the top five in three of the settings and $\mathrm{G}$ (Reminiscence) featured in one.

PDs were collected in the same way for DCM 7 and 8 and are expressed in Table 5 as number per 72 timeframes. With DCM 8, PEs can also be expressed in a similar way which provides an interesting contrast. For all these facilities the numbers of PEs exceeded the numbers of PDs often by a sizeable ratio.

\section{Feedback from mappers and staff focus groups}

No problems were encountered in using DCM 8 to map observations. All time-frames were coded. Feedback from mappers was unanimous that the tool would be easier to use in practice. Reasons given for this were the decrease in complexity of operational rules, lack of degeneration runs, greater clarity over $\mathrm{ME}$ values and clarity over what constitutes a PE. Mappers also appreciated the decision not to code sleep as a +1 , saying that they felt this had elevated the WIB scores of some facilities and then masked subsequent changes in well-being. Mappers also commented positively on the new codes $\mathrm{G}$ (Reminiscence) and $\mathrm{O}$ (attachment to inanimate objects), being able to identify individuals where information about preferences for talking about the past or spending large amounts of time relating to objects, was significant information to feed back to staff teams. There were some concerns that L (Leisure) was now

Table 6. Percentage of time-frames observed in each WIB value/ ME value level (and standard deviation) for DCM 7 and 8

\begin{tabular}{lccc}
\hline $\begin{array}{l}\text { WIB/ME } \\
\text { value }\end{array}$ & DCM 7 & DCM 8 & Pearson correlation \\
\hline+5 & $1.60(3.12)$ & $1.70(3.20)$ & $0.990(p<0.0001)$ \\
+3 & $32.32(20.76)$ & $36.27(19.50)$ & $0.908(p<0.0001)$ \\
+1 & $58.62(18.27)$ & $47.94(16.59)$ & $0.706(p<0.0001)$ \\
-1 & $6.58(9.08)$ & $5.03(8.04)$ & $0.945(p<0.0001)$ \\
-3 & $0.91(4.24)$ & $0.90(4.12)$ & $1.00(p<0.0001)$ \\
-5 & 0 & 0 & - \\
\hline
\end{tabular}

Copyright (C) 2006 John Wiley \& Sons, Ltd. such a large category, particularly in day-care, that it might be easy to lose information about specific preferences. It was agreed that specific preferences would be picked up in notes as it had been in DCM 7. With regards to the new Personal Enhancers, mappers felt that having a balance between the status of the positive and negative was a more constructive way of feeding back information on staff behaviours.

Staff focus groups were also very positive about the changes, particularly the structuring of feedback on Personal Detractions and Personal Enhancers. They appreciated these being linked to the psychological needs of participants. There were many comments that this made the PD feedback make sense in the context of the lives of participants rather than it being seen as a bad mark against staff. Interestingly, a number of staff teams voiced the opinion that they wanted to keep the strong terminology used in the Personal Detraction coding. Many said that it was this list that had made an impression on them when they first learnt about DCM and that it was important not to water this down. There was also a concern that losing degeneration runs would mean that those individuals who spent long periods of time in unattended distress would be missed. It was agreed that long periods of uninterrupted negative states are an indicator of concern and should be highlighted during staff feedback sessions. These are highlighted in feedback of periods of Uninterrupted Negative MEs (UNME) where there has been an uninterrupted run of six time-frames in negative ME values.

The new codes were all received positively as was the decision to record sleep without a ME value.

\section{DISCUSSION}

In summary, concurrent validity has been demonstrated between the scores on DCM 7 and DCM 8 . These similarities are strong enough to suggest that the results from DCM 7 maps can be compared with DCM 8 maps without fear that the differences are due to the revisions of the instrument.

This conclusion should be treated with some caution in that the field trials took place in service settings that have used DCM for a number of years. We deliberately chose this sample as we wanted to have staff and mapper comparative feedback. The sample type may mean, however, that fewer examples of unattended distress would be present than if we had just mapped in facilities that had no prior exposure to DCM. The impact of losing the degeneration rules in DCM 8 may mean that more differences could have been observed in facilities where degeneration would 
have contributed to a much lowered WIB score in DCM 7. Uninterrupted Negative MEs (UNME) were only picked up in one of the facilities mapped here. When comparing DCM 8 data to DCM 7 data containing a lot of degeneration runs, it would be important to take note of the UNMEs in DCM 8.

The interviews with mappers and focus groups with staff teams were very supportive of the changes made in DCM 8 with the overwhelming opinion that the new tool would be more straightforward to use and be a good basis for driving forwards person-centred care practice at an individual and group level. The concerns expressed by mappers and staff groups have been taken forward in the final version of the written material that accompanies DCM 8.

The addition of structured recording of Personal Enhancers was well received in practice. The personal enhancer framework means that mappers can build on the strengths of staff teams rather than by exposure of their weaknesses and failures.

Linking the PDs and PEs to psychological needs made it very clear to staff teams why these aspects of care were so important rather than just seeing them as a reflection of poor attitudes which is how some staff conceptualised the PD list in DCM 7.

In DCM 8, the recording of PDs and PEs is still primarily qualitative in nature. The types of PD and PE are not intended to be used as strictly as the BCC and ME coding. Rather, these frames allow the mapper to engage with the staff group in a reflection on the type of care that is being provided to support people's psychological needs. It is intended to provide a rich vocabulary for describing care.

In some respects, the high correlation between DCM 7 and DCM 8 WIB scores surprised us, given that we had spent so long deliberating on how to refine the observation of well-being and activity. It may be to a certain extent we were simply deconstructing what it is that DCM practitioners observe and making this explicit, rather than redefining what it is we observe. We are also reporting on group results here. At an individual level there may be a significant difference in the WIB scores for those individuals who can get a $+5 \mathrm{ME}$ value through engagement in an activity or with an object that would only have gained $a+3$ in DCM 7. +5 scores at a group level are still relatively rare and would therefore be unlikely to have a significant impact on group scores.

The data reported here is a preliminary validation against DCM 7. It looks as if the data generated by DCM 8 behave in a similar way to DCM 7 and therefore we can assume that the validation of DCM 8 against other measures of quality of life and quality of care would be similar. This is an assumption that requires empirical testing. Likewise, there needs to be a demonstration of inter-rater reliability, test-retest reliability and internal consistency undertaken on DCM 8 data. Strictly speaking, this should be done before DCM 8 is employed for research purposes. Any use of DCM 8 would require the establishment of adequate inter-rater reliability prior to its use by different mappers within a facility whether this was for purposes of practice development or for research. This would also be the case for DCM 7, however.

DCM8 remains a complex tool. It still requires specialist training and checks to maintain reliability in practice. At the commencement of the revision process, it was decided that we were primarily developing a tool that was fit for the development of person-centred care on an individual level. For this reason, it was important to keep the wide variety of codes even though in practice many of these are observed infrequently. The BCC and WIB profiles provides a detailed picture of the percentage of timeframes where behaviours occurred that have potential to enrich the lives of people with dementia and the percentage of time-frames that show behaviours that act against this. Now we have DCM 8 it will be easier to develop shortened forms of DCM that are more fit for research or for inspection purposes.

\section{ACKNOWLEDGEMENTS}

Thanks to all the people at Oxfordshire Mental Healthcare NHS Trust, the Sheffield Care Trust, Norfolk and Waveney Partnership NHS Trust and Wigan Social Services who kindly agreed to participate in this investigation, to all DCM practitioners worldwide who have contributed their experience to the development of DCM 8.

\section{REFERENCES}

Bradford Dementia Group. 1997. Evaluating Dementia Care: The DCM Method, (7th Edn). University of Bradford: Bradford.

Bradford Dementia Group. 2005. DCM 8 User's Manual: The DCM Method, 8th Edition. University of Bradford: Bradford.

Bredin K, Kitwood T, Wattis J. 1995. Decline in quality of life for patients with severe dementia following a ward merger. Int $J$ Geriatr Psychiatry 10: 967-973.

Brooker D. 2005. Dementia Care Mapping (DCM): a review of the research literature. Gerontologist 45(1): 11-18.

Brooker D, Edwards P, Benson S. (eds). 2004. DCM Experience and Insights into Practice. Hawker Publications: London. 
Brooker D, Foster N, Banner A, et al. 1998. The efficacy of Dementia Care Mapping as an audit tool: report of a 3-year British NHS evaluation. Aging Ment Health 2(1): 60-70.

Brooker D, Rogers L (eds). 2001. DCM Think Tank Transcripts 2001. University of Bradford: Bradford.

Dijkstra A, Brown L, Havens B, et al. 2000. An international psychometric testing of the Care Dependency Scale. J Adv Nurs 31(4): 944-952.

Edelman P, Fulton BR, Kuhn D, Chang C. 2005. A comparison of three methods of measuring dementia-specific quality of life: perspectives of residents, staff and observers. Gerontologist 45(1): 27-36.

Fossey J, Lee L, Ballard C. 2002. Dementia Care Mapping as a research tool for measuring quality of life in care settings: psychometric properties. Int J Geriatr Psychiatry 17: 1064-1070.
Kitwood T. 1997. Dementia Reconsidered: The Person Comes First. Open University Press: Buckingham.

Kitwood T, Bredin K. 1994. Charting the course of quality care. $J$ Dement Care 2(3): 22-23.

Kuhn D, Ortigara A, Kasayka R. 2000. Dementia Care Mapping: an innovative tool to measure person-centered care. Alzheim Care Quart 1(3): 7-15.

Surr C, Bonde-Nielsen E. 2003. Inter-rater reliability in DCM. $J$ Dement Care 11(6): 33-36.

Surr C, Brooker D, Edwards P. 2006. Dementia Care Mapping update: DCM8 is ready for action. $J$ Dement Care 14(1): 1719.

Thornton A, Hatton C, Tatham A. 2004. DCM reconsidered: Exploring the reliability and validity of the observational tool. Int J Geriatr Psychiatry 19: 718-726. 